\title{
Response: Proposed Diagnostic Criteria for Definite Isolated Otolith Dysfunction
}

\author{
Myung-Whan Suh ${ }^{1}$ and Toshihisa Murofushi ${ }^{2}$ \\ ${ }^{1}$ Department of Otolaryngology-Head and Neck Surgery, Seoul National University Hospital, Seoul, Korea \\ ${ }^{2}$ Department of Otolaryngology, Teikyo University School of Medicine Mizonokuchi Hospital, Kawasaki, Japan
}

\section{Dear Editor,}

We would like to thank Kenneth Chua Wei De and his colleagues for their valuable comments on, and critique of, our new definition of definite isolated otolith dysfunction (iOD) [1]. As iOD is an emerging concept, it is essential to collect expert opinions when refining the diagnostic criteria. We hope that open discussion and ongoing research will soon yield diagnostic criteria that can achieve international consensus. Here, we reply to the points made by the cited authors and comments that we have received from other experts.

1) We agree that, "the pathognomonic otolith symptoms are not well understood yet and may be easily confused." All of non-spinning, translation, tilt, floating, flipping-over, swaying, or rocking may not be perfect in defining the symptoms of iOD patients. Although iOD symptoms differ from those of typical, peripheral vestibular disorders [2], symptomatic descriptions can mean different things to patients, generalists, physicians, and even otologists [3]. We are especially concerned about the symptomatic overlap between iOD and persistent postural-perceptual dizziness (PPPD) defined by the Barany Society in 2017 [4]. PPPD symptoms may include false or distorted sensations of swaying, rocking, bobbing, or bouncing of oneself (internal non-spinning vertigo) or similar sensation of movements of the surroundings (external non-spinning vertigo) [4]. To overcome the unclear

Received December 14, 2020 / Accepted December 15, 2020

\section{Address for correspondence}

Myung-Whan Suh, MD, PhD

Department of Otorhinolaryngology-Head and Neck Surgery, Seoul National University Hospital, 101 Daehak-ro, Jongno-gu, Seoul 03080, Korea Tel +82-2-2072-4845/ Fax +82-2-766-2447

E-mail drmung@naver.com

This is an Open Access article distributed under the terms of the Creative Commons Attribution Non-Commercial License (https://creativecommons.org/licenses/by-nc/4.0/) which permits unrestricted non-commercial use, distribution, and reproduction in any medium, provided the original work is properly cited. definitions of symptoms, our definition of definite iOD requires laboratory findings indicating utricular and/or saccular dysfunction. We believe that such an objective requirement will allow clinicians and researchers to differentiate PPPD from definite iOD.

2) It is a true that diagnosis of iOD secondary to (co-existing) Meniere's disease (MD) and vestibular migraine (VM) can be challenging. If a patient has another, active, well-defined vestibular disorder, it may be safe to focus on the principal diagnosis because the dizziness may not be caused by otolith dysfunction alone (which is not "isolated" in such patients). We now include the text: "Cannot be explained by another disease or disorder" in our definition. This is a stronger statement than that included in the definition of definite MD by the Barany Society [5], which reads, "Not better accounted for by another vestibular diagnosis." As is the case for patients with definite MD, the diagnosis that better explains the dizziness should be selected when two diagnoses compete or coexist. In a case of definite iOD, any other active vestibular disorder should be the subject of the principal diagnosis, regardless of how effectively iOD explains the dizziness. This is because iOD remains a controversial concept; no strong diagnostic consensus has yet been attained. Also, it is less likely that the cause of dizziness is "isolated" otolith dysfunction in such patients.

However, it should be noted that definite iOD can be diagnosed when the underlying MD and/or VM are/is no longer active. In such cases, the inactive (past) vestibular disorder may be the cause of the present definite iOD (we describe this as "secondary definite iOD").

3) It is true that, "abnormal VEMP may not always suggest a peripheral pathology." Latency abnormality is more often detected in patients with central disorders such as multiple sclerosis [6], vestibular schwannoma [7], stroke [8,9], or migraine [10]. A definite iOD diagnosis requires proof of "abnormal otolith function test: cVEMP, oVEMP, or subjec- 
Table 1. Proposed diagnostic criteria for isolated otolith dysfunction (iOD)

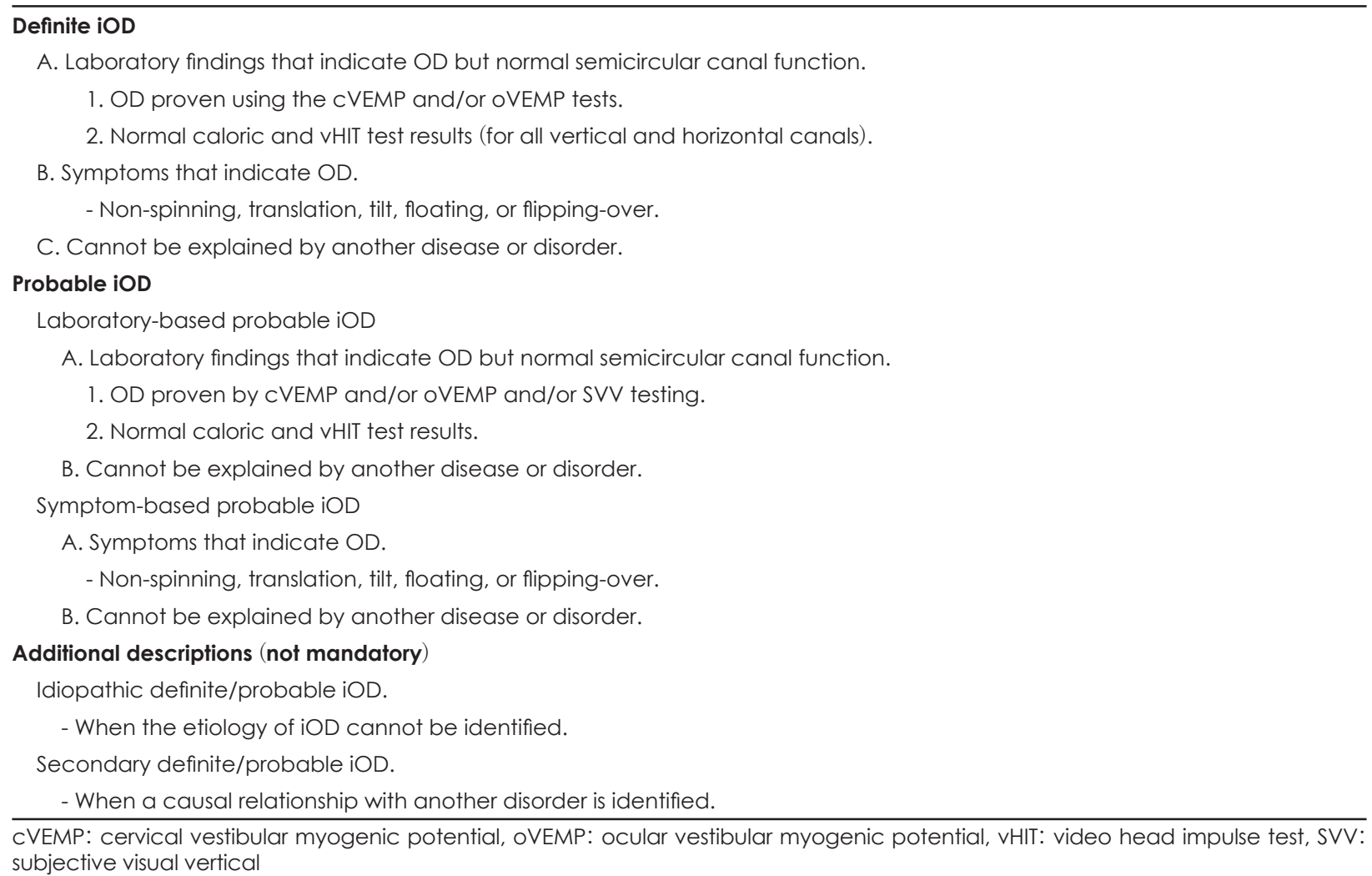

B. Cannot be explained by another disease or disorder.

Symptom-based probable iOD

A. Symptoms that indicate OD.

- Non-spinning, translation, tilt, floating, or flipping-over.

B. Cannot be explained by another disease or disorder.

\section{Additional descriptions (not mandatory)}

Idiopathic definite/probable iOD.

- When the etiology of IOD cannot be identified.

Secondary definite/probable iOD.

- When a causal relationship with another disorder is identified.

cVEMP: cervical vestibular myogenic potential, oVEMP: ocular vestibular myogenic potential, vHIT: video head impulse test, SVV: subjective visual vertical

tive visual vertical (SVV)" [1]. The latency abnormality of VEMP is indicative of a central disorder, not a proof of abnormal otolith function. We have refined our definition of definite iOD to prevent such confusion. Table 4 of our original proposal referred to: "abnormal cVEMP, oVEMP, or SVV" [1]. To reduce confusion, we have changed this to read: "OD proven by cVEMP, oVEMP, or SVV".

Another expert commented that the SVV test might not be optimal in terms of proving otolith dysfunction because the SVV may be affected by central nervous system conditions (including those of the vestibular cortex). Thus, an abnormal SVV may not prove abnormal otolith function in certain patients. SVV may serves as an indicator of probable iOD, but is not the best vestibular evaluation tool. To reflect this fact, we have changed the definition of definite iOD to: "OD proven by cVEMP or oVEMP".

4) We agree that consideration of "whether iOD is of a primary idiopathic cause or secondary" is not mandatory when any causal or co-incidental relationship between IOD and a co-existent disorder is unclear.

5) Indeed, no management plan specific for iOD exists. We hope that our effort to establish an acceptable definition of iOD will lead to creation of such a plan in the future.

6) It is important to clarify that the video head impulse tests must be normal for both the vertical and horizontal canals. iOD can be diagnosed only when all other vestibular organs are normal.

Given the valuable comments that we received, we have revised our iOD definition (Table 1). According to the International Classification of Vestibular Disorders (ICVD) [11], iOD may be a Layer III-A entity (Disorders \& Diseases). Our definition reflects all new ideas suggested up to October 15, 2020. Additional discussion will improve the definition. Also, the Barany society and the ICVD authorities must be approached for comment.

\section{Acknowledgments}

This research was supported by the Korea Health Industry Development Institute (KHDI) of Korean Ministry of Health and Welfare (HI18C0626).

\section{Conflicts of interest}

The authors have no financial conflicts of interest.

\section{ORCID iDs}

Myung-Whan Suh https://orcid.org/0000-0003-1301-2249

Toshihisa Murofushi https://orcid.org/0000-0003-3269-8055

\section{REFERENCES}

1) Park HG, Lee JH, Oh SH, Park MK, Suh MW. Proposal on the di- 
agnostic criteria of definite isolated otolith dysfunction. J Audiol Otol 2019;23:103-11.

2) Murofushi T, Komiyama S, Hayashi Y, Yoshimura E. Frequency preference in cervical vestibular evoked myogenic potential of idiopathic otolithic vertigo patients. Does it reflect otolithic endolymphatic hydrops? Acta Otolaryngol 2015;135:995-9.

3) Bisdorff A, Von Brevern M, Lempert T, Newman-Toker DE. Classification of vestibular symptoms: towards an international classification of vestibular disorders. J Vestib Res 2009;19:1-13.

4) Staab JP, Eckhardt-Henn A, Horii A, Jacob R, Strupp M, Brandt T, et al. Diagnostic criteria for persistent postural-perceptual dizziness (PPPD): consensus document of the committee for the Classification of Vestibular Disorders of the Bárány Society. J Vestib Res 2017;27: 191-208.

5) Lopez-Escamez JA, Carey J, Chung WH, Goebel JA, Magnusson $\mathrm{M}$, Mandalà $\mathrm{M}$, et al. Diagnostic criteria for Menière's disease according to the Classification Committee of the Bárány Society. HNO 2017;65:887-93.
6) Murofushi T, Shimizu K, Takegoshi H, Cheng PW. Diagnostic value of prolonged latencies in the vestibular evoked myogenic potential. Arch Otolaryngol Head Neck Surg 2001;127:1069-72.

7) Lachowska M, Glinka P, Niemczyk K. Air-conducted and skull-tap cervical vestibular evoked myogenic potentials in determining nerve division involvement in vestibular schwannoma patients. Adv Clin Exp Med 2018;27:335-41.

8) Suh MW, Jeong SH, Kim JS. Vestibular evoked myogenic potential: Recording methods and clinical application. J Korean Neurol Assoc 2010;28:1-12.

9) Chen $\mathrm{CH}$, Young $\mathrm{YH}$. Vestibular evoked myogenic potentials in brainstem stroke. Laryngoscope 2003;113:990-3.

10) Baier B, Stieber N, Dieterich M. Vestibular-evoked myogenic potentials in vestibular migraine. J Neurol 2009;256:1447-54.

11) Bisdorff AR, Staab JP, Newman-Toker DE. Overview of the international classification of vestibular disorders. Neurol Clin 2015;33: 54150 , vii. 